\title{
A Time-varying Mixture Memory Multiplicative Error Model
}

\author{
Giovanni De Luca \\ Dipartimento di Studi Aziendali e Quantitativi \\ Università di Napoli Parthenope. \\ Via G. Parisi, 13 - 80133 Napoli, Italy \\ Giampiero M. Gallo \\ New York University in Florence \\ Via dei Bruni, 27 - 50139 Firenze, Italy
}

\begin{abstract}
The dynamics of financial volatility shows a behavior characterized by alternating periods of turbulence and relative quiet. We suggest modelling it as a mixture memory model where time-varying mixing weights are a function of some forcing variable capable of sudden changes. In choosing a mixture approach we rely on previous evidence on the presence of a short-and a long-memory component in the observed series. We apply our model to the main Spanish stock index (IBEX) using the spread between the sovereign national and German bond rates as the forcing variable. The results show a good performance in sample, pointing to the fact that fixed weights may be a limitation to an accurate description of volatility behavior.
\end{abstract}

Keywords: Multiplicative Error Models, Realized Volatility, Mixture Distributions

JEL: C22, C51, C53

\section{Introduction}

In financial econometrics, increasing effort has been devoted to the measurement of volatility of asset prices for the interest it has in risk management, derivative pricing, and asset allocation. While GARCH modeling with daily data has become common among practitioners, in recent times, a lot of work was poured into the potential for empirical applications presented by the availability of intra-daily data. The realized volatility literature has developed techniques for estimating the unobservable quadratic variation of an underlying continuous time process for the evolution of asset prices, thus suggesting a valid alternative to modeling the conditional variance of returns. In what follows we will not dwell on the variety of measures suggested in the literature to take into better consideration phenomena affecting asset price formation such as jumps and microstructure noise, and we adopt as a measure of reference the so-called realized kernel volatility suggested by Barndorff-Nielsen, Hansen, Lunde and Shephard (2008) which is better equipped against these sources of noise.

There is a large debate in the literature about the nature of the high persistence in realized volatility and whether it may be the result of some nonlinearity in the process. The HAR model (Corsi, 2009), although formally not a long-memory model, can reproduce the observed hyperbolic-type decay of the autocorrelation function of (log-)volatility by specifying a sum of volatility components over different horizons. Similarly, a weighted average of past daily realized volatility to predict longer period realized variance is the MIDAS approach of Forsberg and Ghysels (2007). Other authors adopt a logarithmic transformation and apply linear models on the log of realized variance (Forsberg and Ghysels, 2007). Andersen et al. (2007) insert a volatility jump component to capture the abrupt changes characterizing the realized volatility, with significant improvements in the forecasting performance. The intuition by Baillie and Kapetanios (2007) about the existence of both non linear and long memory components in many economics and financial time series is developed by McAleer and Medeiros (2008) who introduce a multiple regime smooth transition extension of the HAR: their model is also able to capture the presence of sign and size asymmetries. Bordignon and Raggi (2012) propose an elegant solution to combine in the same model the non linearity effects, through a Markov switching process, and high persistence, through a fractionally integrated dynamics, capable of improving the accuracy of in- and out-of-sample forecasts. Alternatively, concentrating on long memory explanations, Andersen et al. (2003) suggest a fractionally integrated autoregressive moving average (ARFIMA) model for log-realized volatility; in exchange rate dynamics, Ohanissian et al. (2008) find evidence of long memory as well. However, as noted by Lanne (2006), the ARFIMA model may not be optimal for several reasons: a) a simple short-memory ARMA model can be as good in forecasting the realized volatility of stock returns as a long-memory ARFIMA model (Pong et al., 2004); b) 
The parameters of the FI part and ARMA part can capture similar characteristics (Bos et al., 2002); c) a feasible ARFIMA model must involve a truncation of the infinite-order lag polynomial in practical applications and hence it is an approximation to the "true" model anyway. Corsi et al. (2008) underline how the empirical distributions of ARFIMA and HAR residuals, derived from realized volatility series, tend to exhibit yet unmodeled volatility clustering. In this respect, the presence of regimes (mixture distribution) is also capable of capturing the slowly decaying autocorrelation function of the observed realized volatility series. Maheu and McCurdy (2002, but see also 2007, 2011) find strong statistical evidence of regime changes in both the conditional mean and conditional variance of realized volatility, using a Markov Switching ARMAX representation, where the transition probabilities and the conditional mean of volatility are both functions of the duration of the state. In a GARCH framework, previous contributions had addressed regime switching,cf. the SWARCH model (Hamilton and Susmel, 1994), the MS GARCH model (Dueker, 1997, Klaassen, 2002), and the recent multivariate extensions (Edwards and Susmel, 2003, Higgs and Worthington, 2004, Gallo and Otranto, 2007, 2008). An alternative way to consider changes in regime is given by smooth transition GARCH models (Terasvirta,2009) or other nonlinear models (Terasvirta, 2011). Several other authors indicate the presence of level shifts in GARCH (Perron and Qu, 2010) or breaks in unknown points also in GARCH (He and Maheu, 2010) as the cause of an apparent high persistence. The issue of time varying underlying level of volatility is addressed also by Engle and Rangel (2008), who adapt a spline function in GARCH to capture a low frequency component of volatility (which they connect to macroeconomic factors). Scharth and Medeiros (2009) extend a regression tree model to accommodate smooth splits in regimes controlled by past cumulated returns which account for long-range dependence in volatility.

A different approach favored here is the one adopted by several authors, following the suggestion by Engle (2002; extending the logic of the Autoregressive Conditional Duration (ACD) models of Engle and Russell, 1998) who suggested the use of a Multiplicative Error Model (MEM) for positive valued processes, namely of the product of a conditional expectation that follows a GARCH-type dynamics and a positive valued innovation.

Lanne (2006) has suggested an interesting flexible MEM specification in which the process for realized volatility can be seen as a mixture of two Gamma MEMs with different coefficients for the conditional expectation and different shape parameters for the Gamma (cf. also Ahoniemi and Lanne, 2009).

In this paper, we pursue an alternative to Lanne (2006) by showing that a double conditional expectation model can be improved dropping out the assumption that the two components are both short-memory. In particular, we assume that a short memory component is coupled with a long-memory component and that the probability of observing one of the two components is time-varying. Thus, we extend the mixture approach even further, by adopting a mixture of a shortmemory Gamma MEM and a long-memory Gamma MEM, constructed modelling the conditional expectation as the HAR formulation of Corsi (2009). Moreover, in the application on the Spanish volatility here, the time-varying persistent weights are mainly driven by past deviation of the domestic Government bond rate relative to the German bond rate.

The paper is organized as follows. In Section 2 we summarize some theoretical results on realized volatility in the context of volatility measurement and we summarize some of the features of the Multiplicative Error Model. In Section 3 we present and discuss the models that will be estimated starting from the basic Asymmetric MEM and ending with a formulation including a first component for short-memory features, a second component for long-memory and a specific equation for the dynamics of the mixing weight. Section 4 illustrates the results obtained from the different models applied to the annualized realized volatility of the return rates of the IBEX index. We show that a better fit is obtainable with a mixture memory model when the mixing weight is assumed to be time-varying. Section 5 concludes.

\section{A Multiplicative Error Model for Realized Volatility}

Various arguments can justify the interest in the high-frequency based measures of volatility(cf. various survey papers by Andersen, Bollerslev and Diebold included in the references). Andersen and Bollerslev (1998) pointed out that squared daily returns are a noisy measure of variation: with simulation arguments they show that Mincer-Zarnowitz type regressions of squared returns on any conditional variance forecast would produce a very low R2. Given that volatility or variance of returns is not observed, it has to be substituted with a proxy whose measurement error should vanish under certain conditions. One solution suggested is to refer to the availability of ultra-high frequency data on prices and to compute a variable called realized variance, constructed as

$$
r v_{t}^{2}(\tau)=\sum_{i=1}^{1 / \tau} r_{t-1+i \tau}^{2}=\sum_{i=1}^{1 / \tau}\left(p_{t-1+i \tau}-p_{t-1+(i-1) \tau}\right)^{2}
$$


Where the generic term $r_{-}(\mathrm{t}-1+\mathrm{i} \tau)$ is the return measured intra-daily as the log-price difference of an asset over a (very small) period so that its reciprocal is an integer value, representing the number of intradaily time intervals during the day. When $\tau=1$ we get squared returns back; common choices are fractions of the trading day corresponding to five minutes or thirty minutes intervals. The theoretical support for such an approach stems from the fact that, under suitable conditions, as converges to zero, this measure converges to the integrated variance, that is the integral over a short period of the instantaneous (or spot) volatility of an underlying continuous time diffusion process. Other possible features of the phenomenon could be accommodated, such as the presence of jumps or of market microstructure noise (Barndorff-Nielsen and Shephard, 2002, 2004, 2006; Hansen and Lunde, 2006). In particular, Barndorff-Nielsen, Hansen, Lunde and Shephard (2008) suggest a more refined measure, called realized kernel volatility, in which the daily variance is estimated as:

$$
r v_{t}=\sum_{h=-H}^{H} k\left(\frac{h}{H+1}\right) \sum_{j=|h|+1}^{n} p_{t-1+j} p_{t-1+j-|h|}
$$

In what follows we will consider the square root of realized kernel variance (referred to as realized volatility), a nonnegative valued series. We will model it as a multiplicative process of the form

$$
r v_{t}=\mu_{t} \xi_{t}
$$

Where $\xi_{-} t$ is an iid stochastic process with unit conditional expected value and variance $\phi$, and $\mu_{-} t$ is the conditional expectation of realized volatility. By adopting a GARCH-type structure for $\mu_{-} t$, we get a Multiplicative Error Model to describe the dynamics of the conditional expectation of realized volatility, in its general MEM (q,p) form.

$$
\mu_{t}=\omega+\sum_{j=1}^{q} \alpha_{j} r v_{t-j}+\sum_{i=1}^{p} \beta_{j} \mu_{t-i}
$$

Following Engle and Gallo (2006), it is now standard practice to consider a Gamma specification for $\xi_{-} \mathrm{t}$ with one parameter (as a result of the unit mean constraint): in fact, such a specification turns out to entail the independence of parameter estimates for $\mu$ _ton the value of the shape parameter of the Gamma.

Since a MEM is a generalization of an ACD model (Engle and Russell, 1998), it is natural to extend the specification of the innovation term to accommodate more flexibility and allow for a better fit. As we will see, it is not necessarily an issue of obtaining a model which better predicts the conditional expectation (many different assumptions provide substantially equivalent forecasts), as much as one of having a flexible tool which can adapt to the varying market conditions. We are aiming at a better fit of the density of the distribution altogether, a task which proves useful when we need to derive confidence intervals for expected volatility or evaluate the probability of high values of volatility (say, in a scenario framework).

The models to be compared: common structure and different mixture hypotheses The strategy is to consider a common structure

$$
r v_{t}=\left\{\begin{array}{lll}
\mu_{1 t} \xi_{1 t} & \text { with prob } & \pi_{t} \\
\mu_{2 t} \xi_{2 t} & \text { with prob } & \left(1-\pi_{t}\right)
\end{array}\right.
$$

With a corresponding density function for the innovation

$$
f\left(\frac{r v_{t}}{\mu_{t}} \mid I_{t-1}\right)=\pi_{t} g\left(\frac{r v_{t}}{\mu_{1 t}} \mid I_{t-1} ; \boldsymbol{\theta}_{1}\right)+\left(1-\pi_{t}\right) g\left(\frac{r v_{t}}{\mu_{2 t}} \mid I_{t-1} ; \boldsymbol{\theta}_{2}\right)
$$

Moreover, we define the dummy variable

$$
D_{t}= \begin{cases}1 & r v_{t}<0 \\ 0 & o / \text { wise }\end{cases}
$$

According to the equations of $\mu_{-} 1$ tand $\mu_{-} 2 \mathrm{t}$, the distributional assumptions on $\xi_{-} 1$ tand $\xi_{-} 2 \mathrm{t}$, and the dynamics of $\pi_{-}$twe obtain different models. 


\subsection{The Asymmetric Multiplicative Error Model (AMEM)}

The basic MEM model in its asymmetric version (Engle and Gallo, 2006) is obtained when we do not assume the existence of a mixture $\left(\pi \_t=1\right)$, that is

$$
\mu_{1 t}=\mu_{t}=\omega+\sum_{j=1}^{q} \alpha_{j} r v_{t-j}+\sum_{i=1}^{p} \beta_{j} \mu_{t-i}+\gamma D_{t-1} r v_{t-1}
$$

With

$$
\xi_{1 t}=\xi_{t} \sim G a\left(\lambda, \frac{1}{\lambda}\right)
$$

\subsection{The AMEM with a mixture of innovations}

The second model takes into account the innovations as a mixture of two Gamma distributions. The idea of using a mixture of distributions has a long tradition in the financial literature given its ability to capture the heterogeneity of the market. In an ACD framework De Luca and Gallo (2004) have studied the mixture of two exponential distributions with a fixed mixing weight. From the general formulation a first extension considers two unit-mean Gamma random variables (constrained MAMEM)

$$
\mu_{1 t}=\mu_{2 t}=\mu_{t}=\omega+\sum_{j=1}^{q} \alpha_{j} r v_{t-j}+\sum_{i=1}^{p} \beta_{j} \mu_{t-i}+\gamma D_{t-1} r v_{t-1}
$$

With

$$
\begin{aligned}
& \xi_{1 t} \sim G a\left(\lambda_{1}, \frac{1}{\lambda_{1}}\right) \\
& \xi_{2 t} \sim G a\left(\lambda_{2}, \frac{1}{\lambda_{2}}\right) \\
& \pi_{t}=\pi
\end{aligned}
$$

Such that $E\left(\xi \_1 t\right)=E\left(\xi \_2 t\right)=1$.

The second extension is less restrictive, because it is imposed a unit mean to the mixture, regardless of the expected values of the components of the mixture (unconstrained MAMEM). Then

$$
\mu_{1 t}=\mu_{2 t}=\mu_{t}=\omega+\sum_{j=1}^{q} \alpha_{j} r v_{t-j}+\sum_{i=1}^{p} \beta_{j} \mu_{t-i}+\gamma D_{t-1} r v_{t-1}
$$

With

$$
\begin{aligned}
& \xi_{1 t} \sim G a\left(\lambda_{1}, \gamma_{1}\right) \\
& \xi_{2 t} \sim G a\left(\lambda_{2}, \gamma_{2}\right) \\
& \pi_{t}=\pi
\end{aligned}
$$

The parameter $\gamma \_2$ has to be constrained

$$
\gamma_{2}=\left(1-\pi_{t} \lambda_{1} \gamma_{1}\right) /\left(\left(1-\pi_{t}\right) \lambda_{2}\right)
$$

Such that $\mathrm{E}\left(\xi_{-} \mathrm{t}\right)=1$.

\subsection{The Lanne Model (fixed weights)}

Lanne's (2006) suggestion is to take a mixture of two Gamma densities (with constant weights) and specify two equations with separate coefficients for the conditional expectations. In terms of our notation this latter approach amounts to the following setup 


$$
\begin{aligned}
& \mu_{1 t}=\omega_{1}+\sum_{j=1}^{q} \alpha_{1 j} r v_{t-j}+\sum_{i=1}^{p} \beta_{1 i} \mu_{t-i}+\gamma_{1} D_{t-1} r v_{t-1} \\
& \mu_{2 t}=\omega_{2}+\sum_{j=1}^{q} \alpha_{2 j} r v_{t-j}+\sum_{i=1}^{p} \beta_{2 i} \mu_{t-i}+\gamma_{2} D_{t-1} r v_{t-1}
\end{aligned}
$$

While

$$
\begin{aligned}
& \xi_{1 t} \sim G a\left(\lambda_{1}, \frac{1}{\lambda_{1}}\right) \\
& \xi_{2 t} \sim G a\left(\lambda_{2}, \frac{1}{\lambda_{2}}\right) \\
& \pi_{t}=\pi
\end{aligned}
$$

With a corresponding density function for the innovation

$$
f\left(\frac{r v_{t}}{\mu_{t}} \mid I_{t-1}\right)=\pi g\left(\frac{r v_{t}}{\mu_{1 t}} \mid I_{t-1} ; \boldsymbol{\theta}_{1}\right)+(1-\pi) g\left(\frac{r v_{t}}{\mu_{2 t}} \mid I_{t-1} ; \boldsymbol{\theta}_{2}\right)
$$

Where $\mu \_1$ tand $\mu \_2$ tare the conditional expectations in each of the two regimes and $\xi \_1$ tand $\xi \_$tareGamma random variables with unit expected values. As a result, the overall conditional expectation $\mu$ _titselfcan be seen as a mixture of two conditional expectations.

\subsection{The Mixture Memory MEM (fixed weights)}

We may want to go a step further and extend the Lanne model in two ways. Firstly, we intend to introduce a new equation for $\mu$ 2twhich takes into account the long-memory features of realized volatility. Our idea is that of adopting a realized volatility model including a short-term component represented by an $\operatorname{AMEM}(\mathrm{q}, \mathrm{p})$ formulation and a longmemory component represented by a conditional expected value following a formulation inspired by the Heterogeneous Autoregressive (HAR) equation (Corsi, 2009). We define it Heterogenous Asymmetric MEM (HAMEM) component. The weights of two components are fixed. So wehave

$$
\begin{aligned}
& \mu_{1 t}=\omega_{1}+\sum_{j=1}^{q} \alpha_{1 j} r v_{t-j}+\sum_{i=1}^{p} \beta_{1 i} \mu_{t-i}+\gamma_{1} D_{t-1} r v_{t-1} \\
& \mu_{2 t}=\omega_{1}+\delta_{1} r v_{t-1}+\delta_{2} \sum_{i=1}^{5} r v_{t-i}+\delta_{3} \sum_{i=1}^{22} r v_{t-i}+\gamma_{2} D_{t-1} r v_{t-1}
\end{aligned}
$$

With

$$
\begin{aligned}
& \xi_{1 t} \sim G a\left(\lambda_{1}, \frac{1}{\lambda_{1}}\right) \\
& \xi_{2 t} \sim G a\left(\lambda_{2}, \frac{1}{\lambda_{2}}\right) \\
& \pi_{t}=\pi
\end{aligned}
$$

In the second regime, the expected realized volatility is affected by past realized volatilities at different frequencies. In

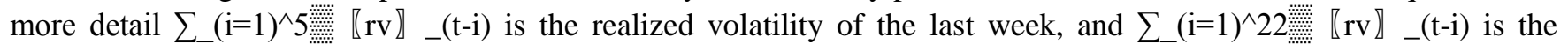
realized volatility of the last month. The dummy term is kept. 


\subsection{The Mixture Memory MEM (time-varying weights)}

The assumption that the mixing proportion $\pi_{-} t$ is a constant may exclude many real situations, so our ultimate proposal is that of introducing a dynamics for $\pi \_t$ :

$$
\begin{aligned}
& \mu_{1 t}=\omega_{1}+\sum_{j=1}^{\bar{q}} \alpha_{1 j} r v_{t-j}+\sum_{i=1}^{p} \beta_{1 i} \mu_{t-i}+\gamma_{1} D_{t-1} r v_{t-1} \\
& \mu_{2 t}=\omega_{1}+\delta_{1} r v_{t-1}+\delta_{2} \sum_{i=1}^{5} r v_{t-i}+\delta_{3} \sum_{i=1}^{22} r v_{t-i}+\gamma_{2} D_{t-1} r v_{t-1}
\end{aligned}
$$

With

$$
\begin{gathered}
\xi_{1 t} \sim G a\left(\lambda_{1}, \frac{1}{\lambda_{1}}\right) \\
\xi_{2 t} \sim G a\left(\lambda_{2}, \frac{1}{\lambda_{2}}\right) \\
\pi_{t}=\Lambda\left(\phi_{0}+\phi_{1} D_{t-1} r v_{t-1}+\phi_{2} S P R E A D_{[t-1]}+\phi \pi_{t-1}\right)
\end{gathered}
$$

Where

$$
\Lambda(x)=\frac{\exp (x)}{1+\exp (x)}
$$

In the logistic equation of $\pi_{-} t$ we include the one-lagged realized volatility multiplied by the one-lagged dummy variable assuming value 1 if the one-lagged return is negative, the variable 『SPREAD】_([t-1])which denotes the value of the spread between government and German bond yields at the last available time before time $t$, and finally an autoregressive term of the weight. In the presence of a negative coefficient $\phi_{-} 2$, a higher spread involves a lower value of $\pi_{-} t$, which implies more weight to the long-memory component of the volatility. For sake of comparison we also consider the Lanne model with time-varying weights following the above dynamics.

\section{Real data analysis}

In this application, we consider the daily annualized realized volatility (5-minute) of IBEX35 index, the benchmark stock market index of the Madrid stock exchange, from Jan. 2, 2002 to Dec. 30, 2011 (http://realized.oxfordman.ox.ac.uk).

Figure 4 displays the plot of the annualized realized volatility and the estimated autocorrelation function is drawn up to lag 50. A high persistent nature of the realized volatility is observed.

A short memory model which does not take into account this feature can be misleading in fitting and forecasting the series.

Tables 1-4 report the estimates of the models described in the previous section. The first table contains the estimates of the basic AMEM, with and without a mixture of distributions assumption. It is clear that the mixture hypothesis remarkably increases the fit of the model, as we can see looking at the mean log-likelihood and at the Akaike Information Criterion (AIC), in particular when the hypothesis of unit mean on the two components of the mixture is replaced by the hypothesis of unit mean on the mixture.

In Table 2, two Lanne models are estimated with different orders for the second component. They offer a better fit with respect to the basic models, and in particular the formulation withp $=\mathrm{q}=1$ for the second component is slightly better in terms of AIC. Note that the asymmetric component is significant in both the components in both the models.

Then, we introduce the mixture memory models. The first case refers to a fixed weight scheme. For the second component we consider both a model where the last term is the realized volatility of the last week (5 days) and a model where the last term is the realized volatility of the last month (22 days). The results are good but, surprisingly, not superior to the Lanne models. As suggested by Lanne (2006), although the mixture-MEM model is a short-memory model, the parameter estimates can produce rather slowly decaying autocorrelation functions. 
Finally, let us illustrate the behavior of two time-varying weights models. We have selected the best Lanne model, that is the model with $\operatorname{AMEM}(2,1)$ and $\operatorname{AMEM}(1,1)$ components, and the best mixture memory model, the $\operatorname{HMEM}(22)$. The estimates are reported in table 4. The Akaike information criterion shows a clear improvement compared to fixed weights model. In particular, the mixture memory model has the lower AIC. The dynamics of the estimated timevarying mixing weight appears to be quite erratic (see Figure 4). What is evident is that periods of high volatility are strongly associated with low values of $\pi$ t (we report that the correlation coefficient between the two variables is equal to -0.65 , without claiming, however, the linearity of the relationship). This means that the increase of volatility reduces the weight of the short-memory component of the mixture in favor of the long-memory component.

Figures 2 and 3 report the observed and fitted realized volatility for the simple $\operatorname{AMEM}(2,1)$ and for the most effective model, the mixture memory model with time-varying weights. Moreover, it is also drawn their differences, that is 『rv 』_t-(rv)^t. We can observe that for the basic model considered the differences tend to have a positive average, and that positive differences are much more stronger than negative differences. In second case, the average difference is null, and some positive peaks observed in the basic model tend to be less pronounced. This means that the last formulation is able to capture in a better way the peaks of volatility.

We have performed an extensive one-step-ahead prediction analysis carrying out 252 recursive estimates of the models. In detail, given a model, we have initially considered the observations from the beginning to 2010/12/31 (check), so to obtain the prediction of the annualized realized volatility one-day-ahead. Then we have added one observation to the sample, we have reestimated the model and computed next one-step-ahead prediction, and so on until the last prediction at 2011/12/31 (check). The models considered are:

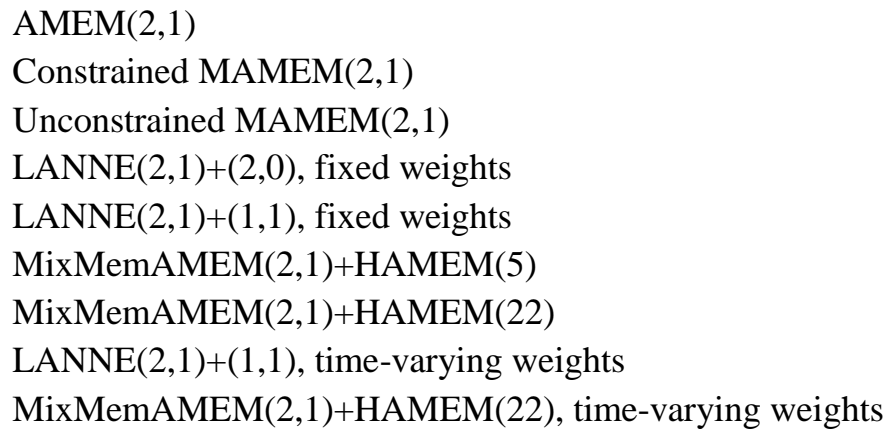

The computation of the root mean squared error (Table 5) favors the last model in a clear way. The mixture memory with the short-memory component represented by the AMEM model and the long-memory component represented by the HAMEM, with time-varying mixing weights, is the model which provides the best one-day-ahead forecasts. Future research will examine the h-step-ahead predictions $(\mathrm{h}>1)$.

1. $\operatorname{AMEM}(2,1)$

2. Constrained MAMEM(2,1)

3. Unconstrained MAMEM $(2,1)$

4. LANNE(2,1)+(2,0), fixed weights

5. LANNE(2,1)+(1,1), fixed weights

6. MixMemAMEM(2,1)+HAMEM(5)

7. MixMemAMEM(2,1)+HAMEM(22)

8. $\operatorname{LANNE}(2,1)+(1,1)$, time-varying weights

9. MixMemAMEM(2,1)+HAMEM(22), time-varying weights 
Table 1: Basic Asymmetric Multiplicative Error Models (standard errors in brackets).

\begin{tabular}{cccc}
\hline Parameter & $\operatorname{AMEM}(2,1)$ & $\begin{array}{c}\text { Constrained } \\
\text { MAMEM }(2,1)\end{array}$ & Unconstrained MAMEM $(2,1)$ \\
\hline$\omega$ & $0.0021(0.0004)$ & $0.0013(0.0003)$ & $0.0012(0.0003)$ \\
$\alpha_{1}$ & $0.4191(0.0224)$ & $0.3988(0.0216)$ & $0.3855(0.0217)$ \\
$\alpha_{2}$ & $-0.2144(0.0338)$ & $-0.2259(0.0310)$ & $-0.2220(0.0308)$ \\
$\beta_{1}$ & $0.7548(0.0256)$ & $0.7939(0.0220)$ & $0.8057(0.0213)$ \\
$\gamma$ & $0.0524(0.0071)$ & $0.0450(0.0061)$ & $0.0448(0.0061)$ \\
\hline$\pi_{1}$ & - & $0.9214(0.0237)$ & $0.8854(0.0298)$ \\
$\lambda_{1}$ & $0.0540(0.0015)$ & $0.0390(0.0020)$ & $0.0352(0.0021)$ \\
$\lambda_{2}$ & - & $0.2282(0.0438)$ & $0.1785(0.0280)$ \\
$\gamma_{1}$ & - & - & $27.6548(1.5826)$ \\
\hline Mean log-lik & 1.9736 & 2.0075 & 2.0165 \\
AIC & -3.9425 & -4.0087 & -4.0258 \\
\hline
\end{tabular}

Table 2: Lanne models, fixed weights (standard errors in brackets).

\begin{tabular}{ccc}
\hline Parameter & $\operatorname{AMEM}(2,1)+\operatorname{AMEM}(2,0)$ & $\operatorname{AMEM}(2,1)+\operatorname{AMEM}(1,1)$ \\
\hline$\omega_{1}$ & $0.0007(0.0002)$ & $0.0006(0.0002)$ \\
$\alpha_{11}$ & $0.3227(0.0269)$ & $0.3287(0.0278)$ \\
$\alpha_{12}$ & $-0.1853(0.0331)$ & $-0.1951(0.0343)$ \\
$\beta_{1}$ & $0.8316(0.0202)$ & $0.8379(0.0203)$ \\
$\gamma_{1}$ & $0.0409(0.0060)$ & $0.0385(0.0062)$ \\
$\omega_{2}$ & $0.0303(0.0071)$ & $0.0225(0.0068)$ \\
$\alpha_{121}$ & $0.7135(0.1062)$ & $0.6255(0.1155)$ \\
$\alpha_{22}$ & $0.1582(0.1006)$ & - \\
$\beta_{2}$ & - & $0.2462(0.1095)$ \\
$\gamma_{2}$ & $0.1442(0.0495)$ & $0.1663(0.0507)$ \\
$\pi_{1}$ & $0.8276(0.0349)$ & $0.8200(0.0371)$ \\
\hline$\lambda_{1}$ & $0.0335(0.0019)$ & $0.0332(0.0020)$ \\
$\lambda_{2}$ & $0.1064(0.0117)$ & $0.1048(0.0114)$ \\
\hline Mean log-lik & 2.0240 & 2.0245 \\
AIC & -4.0385 & -4.0394 \\
\hline
\end{tabular}

Table 3: Mixture memory realized volatility models with AMEM(2,1) and HAMEM(r) components, fixed weights (standard errors in brackets).

\begin{tabular}{ccc}
\hline Parameter & $r=5$ & $r=22$ \\
\hline$\omega_{1}$ & $0.0007(0.0002)$ & $0.0006(0.0002)$ \\
$\alpha_{11}$ & $0.3234(0.0268)$ & $0.3333(0.0259)$ \\
$\alpha_{12}$ & $-0.1835(0.0335)$ & $-0.2171(0.0337)$ \\
$\beta_{1}$ & $0.8287(0.0208)$ & $0.8568(0.0214)$ \\
$\gamma_{1}$ & $0.0413(0.0061)$ & $0.0366(0.0059)$ \\
\hline$\omega_{2}$ & $0.0307(0.0077)$ & $0.0368(0.0090)$ \\
$\delta_{1}$ & $0.7196(0.1314)$ & $0.6447(0.1436)$ \\
$\delta_{2}$ & $0.0311(0.0272)$ & $0.1101(0.0520)$ \\
$\delta_{3}$ & & $-0.0162(0.0093)$ \\
$\gamma_{2}$ & $0.1408(0.0505)$ & $0.1422(0.0519)$ \\
\hline$\pi$ & $0.8320(0.0356)$ & $0.8417(0.0339)$ \\
\hline$\lambda_{1}$ & $0.0337(0.0020)$ & $0.0343(0.0020)$ \\
$\lambda_{2}$ & $0.1076(0.0119)$ & $0.1043(0.0119)$ \\
\hline Mean log-lik & 2.0234 & 2.0245
\end{tabular}




\begin{tabular}{ccc} 
AIC & -4.0381 & -4.0387 \\
\hline
\end{tabular}

Table 4: Lanne model with $\operatorname{AMEM}(2,1)$ and $\operatorname{AMEM}(1,1)$ components, time-varying weigths. Mixture memory realized volatility models with $\operatorname{AMEM}(2,1)$ and HAMEM(22) components, time-varying weights (standard errors in brackets).

\begin{tabular}{ccc}
\hline Parameter & Lanne model & Mixture memory model \\
\hline$\omega_{1}$ & $0.0008(0.0002)$ & $0.0007(0.0002)$ \\
$\alpha_{11}$ & $0.3135(0.0245)$ & $0.3156(0.0243)$ \\
$\alpha_{12}$ & $-0.1692(0.0308)$ & $-0.1945(0.0309)$ \\
$\beta_{1}$ & $0.8251(0.0194)$ & $0.8518(0.0201)$ \\
$\gamma_{1}$ & $0.0373(0.0058)$ & $0.0331(0.0056)$ \\
$\omega_{2}$ & $0.0496(0.0097)$ & $0.0552(0.0107)$ \\
$\alpha_{21}$ & $0.7299(0.0990)$ & - \\
$\beta_{2}$ & $0.0694(0.0979)$ & - \\
$\delta_{1}$ & - & $0.6396(0.1082)$ \\
$\delta_{2}$ & - & $0.0846(0.0361)$ \\
$\delta_{3}$ & - & $-0.0134(0.0064)$ \\
$\gamma_{2}$ & $0.1203(0.0471)$ & $0.1079(0.0494)$ \\
\hline$\phi_{0}$ & $-0.7700(0.5636)$ & $0.1313(1.6921)$ \\
$\phi_{1}$ & $-2.8951(0.9028)$ & $-3.5434(1.1879)$ \\
$\phi_{2}$ & $-0.1688(0.0729)$ & $-0.2838(0.2191)$ \\
$\phi$ & $3.4268(0.6033)$ & $2.5205(1.8522)$ \\
\hline$\lambda_{1}$ & $0.0341(0.0017)$ & $0.0342(0.0017)$ \\
$\lambda_{2}$ & $0.0969(0.0102)$ & $0.0902(0.0098)$ \\
\hline Mean log-lik & 2.0235 & 2.0337 \\
AIC & -4.0532 & -4.0547 \\
\hline
\end{tabular}

Figure 1: Ibex - Annualized realized volatility ( $5 \mathrm{~min}$ ) and estimated autocorrelation function 

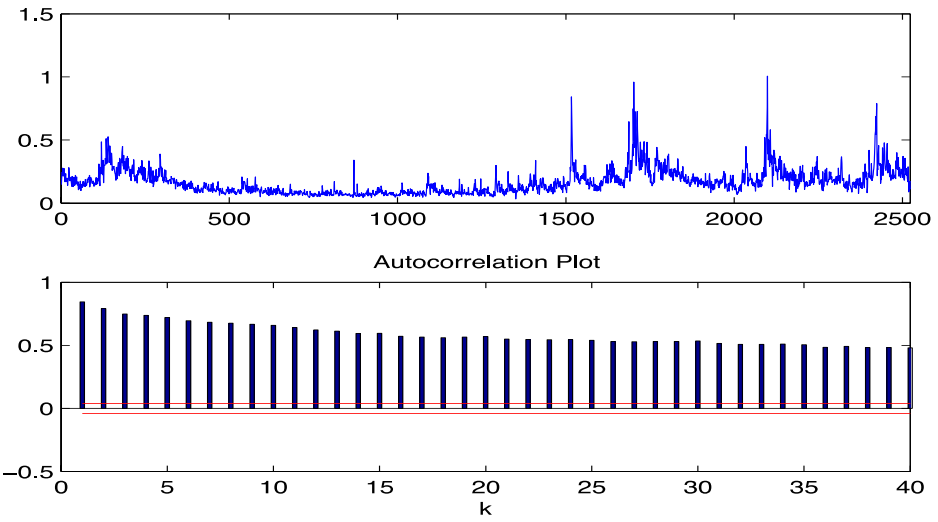

Figure 2: Top: IBEX annualized realized volatility 『rv】_t(blue) and fitted realized volatility (rv)^_t (red) from the $\operatorname{AMEM}(2,1)$ model. Bottom: \rv】_t-(rv)^_t.
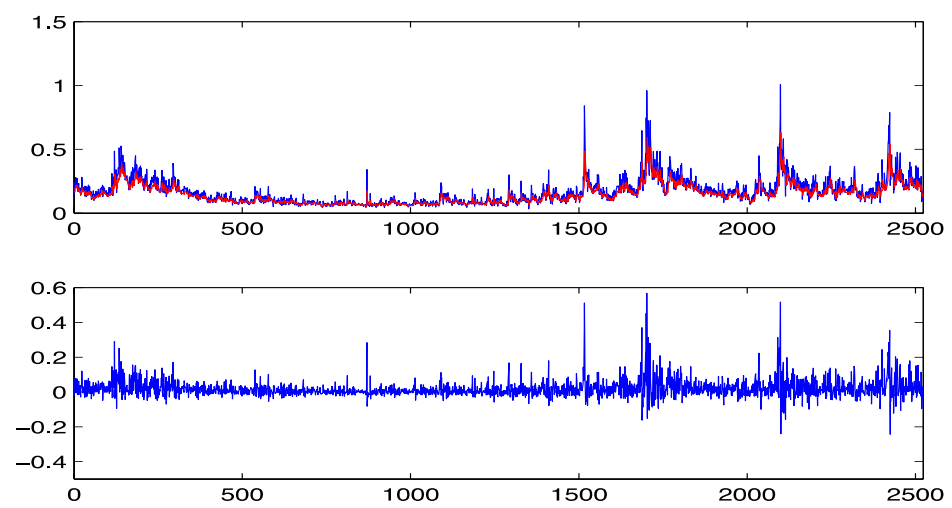

Figure 3: Top: IBEX annualized realized volatility $\llbracket r v \rrbracket \_t$ (blue) and fitted realized volatility (rv)^_t (red) from

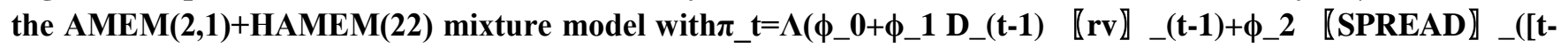

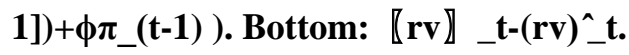



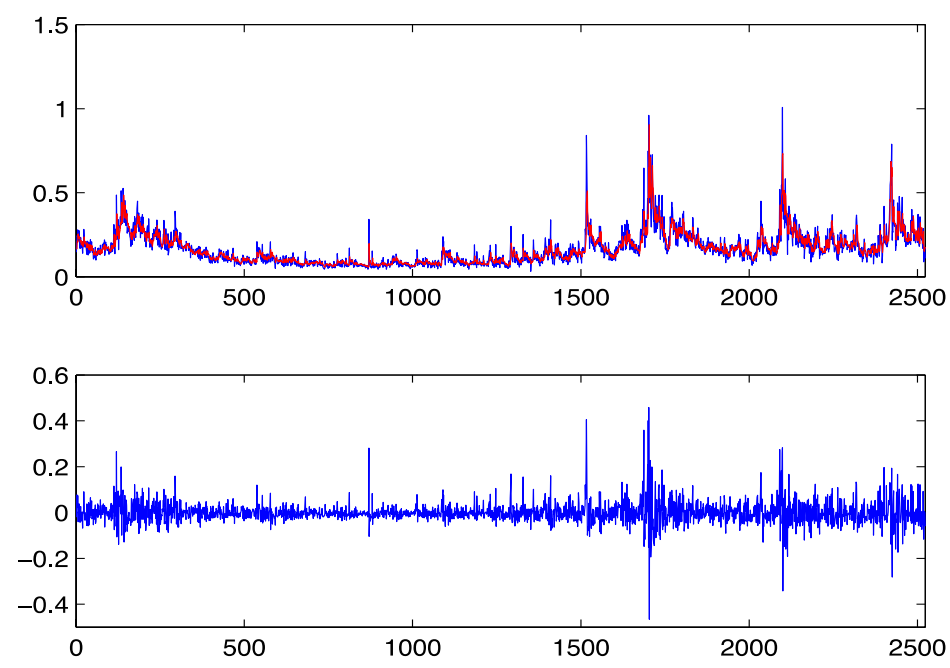

Figure 4: Top: IBEX annualized realized volatility \rv》_t. Bottom: the estimated mixing weight $\pi_{-}$ffor the time-varying mixture memory model.
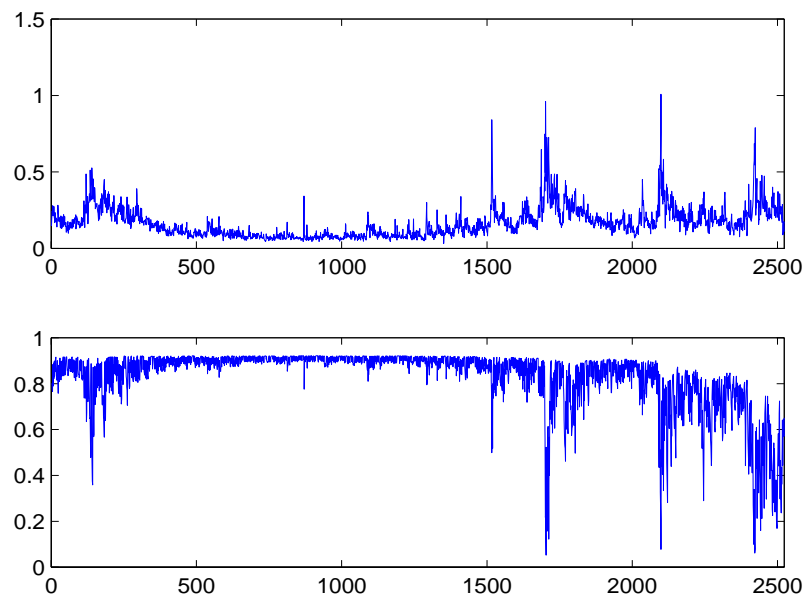

Table 5: Mean-squared error for 252 one-step-ahead prediction of annualized realized volatility

\begin{tabular}{|l|c|}
\hline \multicolumn{1}{|c|}{ Model } & Meansquared error \\
\hline AMEM $(2,1)$ & 0.0039 \\
\hline ConstrainedMAMEM $(2,1)$ & 0.0040 \\
\hline UnconstrainedMAMEM $(2,1)$ & 0.0040 \\
\hline LANNE(2,1)+(1,1) & 0.0039 \\
\hline MMAMEM(2,1)+HAMEM(22) & 0.0040 \\
\hline LANNE(2,1)+(1,1)tv & 0.0039 \\
\hline MMAMEM(2,1)+HAMEM(22)tv & 0.0036 \\
\hline
\end{tabular}

\section{Concluding Remarks}

In this paper, we have introduced a mixture-memory specification for the analysis of realized volatility which takes into account both a short-memory and a long-memory component. The short-memory component is represented by an $\operatorname{AMEM}(2,1)$ while the long-memory part is given by a formulation that resembles the Heterogenous Autoregressive model of Corsi (2009). The novel formulation is estimated under two hypotheses about the weights. We first assume fixed weights, obtaining results which are substantially the same as the benchmark Lanne model. We then propose some richer dynamics for the time-varying mixing weights is proposed, where the latter are assumed to be a function of some forcing variable. In our application on the Spanish reference index IBEX, we chose such a variable to be the 
lagged one spread between the Spanish Bonos and the German Bund yields. We get a strong improvement in terms of in sample performance with better fit and diagnostics than the benchmark. In one step-ahead forecasting, our model has a mean square error which is substantially lower than the benchmark and our previous suggestion. The final comment is that the turmoil originating in the bond market when the Southern European countries came under attack with fears of a severe crisis involving the Euro is a relevant determinant of which dynamics rules stock market volatility.

\section{References}

Ahoniemi K. and Lanne M. (2009), Joint Modelling of Call and Put Implied Volatility, International Journal of Forecasting, 25, 239-258.

Andersen T.G. and Bollerslev T. (1997), Intraday Periodicity and Volatility Persistence in Financial Markets,Journal of Empirical Finance, 4, 115-158.

Andersen T.G. and Bollerslev T. (1998), Answering the Skeptics: Yes, Standard Volatility Models Do Provide Accurate Forecasts, International Economic Review, 39, 885-905.

Andersen T.G., Bollerslev T., Christoffersen P.F., and Diebold,F.X., (2006), Volatility and Correlation Forecasting, in G. Elliott, C.W.J. Granger, and Timmermann,A. (eds.), Handbook of Economic Forecasting. Amsterdam: North-Holland.

Andersen T.G., Bollerslev T.,DieboldF.X., and LabysP. (2000), Great Realizations, Risk, 13, 105-108.

Andersen T.G., BollerslevT., DieboldF.X., and Labys P. (2003), Modeling and Forecasting Realized Volatility,Econometrica, 71, 579-625.

Andersen T.G., BollerslevT., and Diebold,F.X. (2007), Roughing It Up: Including Jump Components in the Measurement, Modeling, and Forecasting of Return Volatility, The Review of Economics and Statistics, 89, 701-720.

Andersen, T.G., BollerslevT., and DieboldF.X. (2010), Parametric and Nonparametric Volatility Measurement. In Y. Ait-Sahalia and L.P. Hansen (eds.), Handbook of Financial Econometrics, Amsterdam: North-Holland.

Bandorff-Nielsen O.E. and Shephard N. (2002), Econometric analysis of realized volatility and its use in estimating stochastic volatility models,Journal of the Royal Statistical Society, Series B, 64, 253-280.

Bandorff-Nielsen O.E. and Shephard N. (2004), Power and bipower variation with stochastic volatility and jumps (with discussion),Journal of Financial Econometrics, 2, 1-48.

Bandorff-Nielsen O.E. and Shephard N. (2006), Econometrics of Testing for Jumps in Financial Economics Using Bipower Variation, Journal of Financial Econometrics, 4, 1-30.

Barndorff-Nielsen O.E., Hansen P.R.,Lunde A.and ShephardN.(2008), Designing Realised Kernels to Measure the Expost Variation of Equity Prices in the Presence of Noise, Econometrica, 76, 1481-1536.

Bordignon S. and Raggi D. (2012), Long Memory and nonlinearities in realized volatility: a Markov switching approach,Computational Statistics and Data Analysis, 56, 3730- 3742.

Bos C.S., FransesP.H. and OomsM.(2002), Inflation Forecast Intervals and Long Memory Regression Models,International Journal of Forecasting, 18, 243-264.

Corsi F. (2009), A Simple Approximate Long-Memory Model of Realized Volatility. Journal of Financial Econometrics, 7, 174-196.

Corsi F., MittnikS., PigorschC., and PigorschU. (2008), The Volatility of Realized Volatility, Econometric Reviews, $27,46-78$.

De Luca G. and GalloG.M. (2004), Mixture Processes for Financial Intradaily Durations, Studies in Nonlinear Dynamics and Econometrics, 8.

Dueker M.J. (1997), Markov Switching in GARCH Processes and Mean-reverting Stock-market Volatility,Journal of Business and Economic Statistics, 15, 26-34.

Edwards S. and SusmelR. (2003), Interest-rate Volatility in Emerging Markets, The Review of Economics and Statistics, 85, 328-348.

Engle R.F. (2002), New Frontiers for ARCH Models, Journal of Applied Econometrics, 17, 425-446.

Engle R.F. and GalloG.M. (2006), A Multiple Indicators Model for Volatility Using Intra-daily Data, Journal of Econometrics, 131, 3-27.

Engle R.F. and RangelJ.G. (2008), The Spline-GARCH Model for Low-frequency Volatility and its Global Macroeconomic Causes, Review of Financial Studies, 21, 1187- 1222.

Engle R.F. and Russell J.R. (1998), Autoregressive Conditional Duration: a new model for irregularly spaced transaction data, Econometrica, 66, 1127-1162.

Forsberg L. and Ghysels E. (2007), Why Do Absolute Returns Predict Volatility So Well? Journal of Financial Econometrics, 5, 31-67. 
Gallo G.M. and OtrantoE. (2007), Volatility Transmission across Markets: a Multichain Markov Switching Model, Applied Financial Economics, 17, 659-670.

Gallo G.M. and OtrantoE. (2008), Volatility Spillovers, Interdependence and Comovements: a Markov Switching Approach, Computational Statistics and Data Analysis, 52, 3011-3026.

Hamilton J.D. and SusmelR. (1994), Autoregressive Conditional Heteroskedasticity and Changes in Regime, Journal of Econometrics, 64, 307-333.

Hansen P.R. and Lunde A. (2006), Realized Variance and Market Microstructure Noise (with discussion), Journal of Business and Economic Statistics, 24, 127-161.

He Z. and MaheuJ.M. (2010), Real Time Detection of Structural Breaks in GARCH Models, Computational Statistics and Data Analysis, 54, 2628-2640.

Higgs H. and WorthingtonA.C. (2004), Transmission of Returns and Volatility in Art Markets: a Multivariate GARCH Analysis, Applied Economics Letters, 11, 217-222.

Klaassen F. (2002), Improving GARCH Volatility Forecasts with Regime-Switching GARCH, Empirical Economics, 27, 363-394.

Lanne, M. 2006. A Mixture Multiplicative Error Model for Realized Volatility. Journal of Financial Econometrics 4, 594-616.

Maheu J.M. and McCurdyT.H. (2002), Non-linear Features of Realized FX Volatility, Review of Economics and Statistics, 84, 668-681.

Maheu J.M. and McCurdyT.H. (2007), Components of market risk and return, Journal of Financial Econometrics, 5, $560-590$.

Maheu J.M. and McCurdyT.H. (2011), Do high-frequency measures of volatility improve forecasts of return distributions? Journal of Econometrics, 160, 69-76.

McAleer M. and MedeirosM.C.(2008), A Multiple Regime Smooth Transition Heterogeneous Autoregressive Model for Long Memory and Asymmetries, Journal of Econometrics, 147, 104-119.

Ohanissian A., RussellJ. and TsayT. (2008), True or Spurious Long Memory? A New Test, Journal of Business and Economic Statistics, 26, 161-175.

Perron, B., and Z. Qu. 2010. Long-memory and Level Shifts in the Volatility of Stock Market Return Indices. Journal of Business and Economic Statistics 28, 275-290

Pong, S., M.B. Shackleton, S.J. Taylor, and X. Xu. 2004. Forecasting Currency Volatility: a Comparison of Implied Volatilities and AR(FI)MA Models. Journal of Banking and Finance 28, 2541-2563.

ScharthM. and Medeiros M. (2009), Asymmetric Effects and Long Memory in the Volatility of DJIA Stocks,International Journal of Forecasting, 25, 304-327.

Terasvirta, T. (2009), An Introduction to Univariate GARCH Models. In T.G. Andersen, R.A. Davis, J.-P. Kreiß and T. Mikosch (eds.), Handbook of Financial Time Series, 17-42, Berlin-Heidelberg: Springer.

Terasvirta, T. (2011), Nonlinear Models for Autoregressive Conditional Heteroskedasticity. CREATES Research Papers 2011-02, School of Economics and Management, University of Aarhus. 